\title{
Una revisión de los condicionantes de la política exterior latinoamericana: dimensiones de análisis para examinar la relación entre lo "interno" y lo "externo" desde la periferia argentina
}

\author{
María Cecilia Míguez*
}

\section{Resumen}

Este artículo formaliza un esquema de dimensiones de análisis para la interpretación de la política exterior en un país periférico. Retoma tradiciones de las Relaciones Internacionales (RI) para poner en discusión algunas de ellas, y para rescatar la importancia de los estudios críticos e históricos como fuente de la reflexión conceptual. Analiza, en especial, los denominados condicionantes domésticos de la política exterior, los desagrega, para luego reivindicar la importancia de una historia total y de una interpretación más compleja de las fronteras entre "lo interno" y "lo externo". El esquema analítico se relaciona con los siguientes objetivos: en primer lugar, recorrer brevemente las escuelas de pensamiento que han abordado la problemática de los condicionantes internos de la política exterior. Luego, desagregar las dimensiones de análisis para interpretar esos condicionantes, pero a la luz de una clara teoría del estado, de una corriente crítica y de un enfoque histórico de las relaciones internacionales. En tercer término, es intención de este estudio complejizar la distinción entre factores internos y externos, teniendo en cuenta distintos niveles de análisis donde ambos se encuentran profundamente relacionados.

Palabras clave: Análisis de Política Exterior- Argentina- América Latina- Condicionantes internos

\begin{abstract}
This article formalizes a scheme of variables for the interpretation and analysis of foreign policy in a peripheral country. It takes up traditions of International Relations (IR) to discuss some of them, and to rescue the importance of critical and historical studies as a source of conceptual reflection. It analyzes, in particular, the so-called domestic determinants of foreign policy, disaggregates them, and then claims the importance of a total history and a more complex interpretation of the boundaries between "internal" and "external". The analytical scheme is related to the following objectives: first, to briefly review the schools of thought that have addressed the issue of the internal determinants of foreign policy. Then, to disaggregate the variables for interpreting internal determinants, but in the light of a clear theory of the state, a critical current and a historical approach to international relations. Thirdly, it is the intention of this study to make the distinction between internal and external factors more complex, taking into account different levels of analysis where both are deeply related.
\end{abstract}

Key words: Foreign policy analysis- Argentina- Latin America- Domestic determinants

TRABAJO RECIBIDO: 15/06/2021 TRABAJO ACEPTADO: 11/11/2021

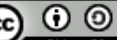

Esta obra está bajo una licencia internacional https://creativecommons.org/licenses/by-sa/4.0/

\footnotetext{
* Investigadora de CONICET, Vicedirectora del Instituto de Estudios Históricos Económicos Sociales e Internacionales (IDEHESI). Profesora Adjunta en la Universidad de Buenos Aires. E-mail: mmccmiguez@gmail.com
} 


\section{Introducción}

Este trabajo pretende formalizar un esquema con dimensiones de análisis para la interpretación y análisis de la política exterior en un país periférico. Rescata la importancia de los estudios críticos e históricos como fuente de la reflexión conceptual. Analiza, en especial, los denominados condicionantes domésticos de la política exterior, los desagrega, para luego reivindicar la importancia de una historia total y de una interpretación más compleja de las fronteras entre "lo interno" y "lo externo".

El estudio de la vinculación entre las denominadas variables internas y externas de la política exterior tiene ya una larga tradición en la disciplina de las RI. Desde la década de 1950 en adelante varias corrientes han desarrollado enfoques y perspectivas para abordar la cuestión de las condiciones domésticas de la política internacional. Las primeras de ellas surgieron en los Estados Unidos, dando origen a distintos modelos de Análisis de Política Exterior (APE). Algunos de esos enfoques que suelen utilizarse en forma acrítica en la periferia, son profundamente estado-céntricos, otros sistémicos, algunos construyen tipos ideales ahistóricos, otros se basan en el individualismo metodológico, entre otros aspectos. Lo cierto es que dichos modelos se vuelven insuficientes a la hora de explicar la política de los Estados latinoamericanos -signada por relaciones de dependencia- donde el entramado entre lo interno y lo externo es particular ${ }^{1}$.

La condición estructural de dependencia que impacta en el desarrollo de los países de Latinoamérica, y en el comportamiento de sus clases dirigentes, élites económicas, intelectuales y políticas, ha sido largamente estudiada por las denominadas Teorías de la Dependencia (Dos Santos, 2003). Al mismo tiempo, este artículo parte de las corrientes que consideran que dicha condición continúa vigente (Taush, 2010) pero que ello explica en su totalidad la compleja combinación e imbricación de elementos internos y externos que nos permiten interpretar una política exterior. A diferencia de algunas visiones estructuralistas de las Teorías de la Dependencia, se e considera aquí que, partiendo de determinada configuración del sistema internacional, los condicionantes internos de orden político, económico y social constituyen variables centrales para explicar la política exterior que no deben ser descuidadas. En el plano interno se definen las disputas y contradicciones que se expresan en la adopción de determinada política exterior, aunque muchas de ellas provengan de dinámicas transnacionales; y al mismo tiempo es en esa misma articulación donde es posible elaborar estrategias políticas para atender las necesidades de grandes mayorías.

El desdibujamiento de las fronteras entre lo interno y lo externo es una experiencia concreta e histórica en nuestra América Latina, tal como lo han señalado muchísimos autores. Dicho desdibujamiento se comprueba en distinta medida para todas las naciones. La Historia de las Relaciones Internacionales lo reflejó en sus trabajos empíricos y analíticos (Renouvin et Duroselle, 2010) (Frank, 2012). El proceso de globalización -en el marco del auge del neoliberalismo de las últimas décadas del siglo XX- hizo aún más lábiles esas fronteras, dando protagonismo a una multiplicidad de actores en el escenario de la política internacional. Ya desde sus primeros trabajos, Keohane y Nye (1974) teorizaron sobre las relaciones transnacionales y la necesidad de incluir nuevos escenarios y protagonismos en la teoría. Las corrientes de la Economía Política Internacional también contribuyeron a la incorporación de nuevos actores de las relaciones internacionales, más allá de los Estados. Los estudios trasnacionales y globales, en sus diversas escuelas, han sido prolíficos en este sentido ${ }^{2}$.

\footnotetext{
${ }^{1}$ En este artículo partimos de considerar que la dependencia es una característica de las formaciones sociales latinoamericanas (Marini, 2008) que continúa vigente en la actualidad, más allá de las transformaciones del capitalismo global (Farny, 2016).
} 
Pero el Estado continúa siendo la llave que orienta la estrategia de inserción internacional a través de sus políticas, promoviendo, profundizando o desalentando condiciones históricas de vinculación con el sistema mundial ${ }^{3}$. Y la decisión política también está condicionada por factores internos que es necesario distinguir. Por esa razón, se asume aquí que es necesaria la distinción analítica de lo interno -aquello que sucede en el plano de las fronteras del estado nación- y lo externo, que involucra la relación con países y actores por fuera de dicho plano, pero siempre teniendo en cuenta el carácter dialéctico y complejo de dicha distinción que buscaremos matizar.

Existen en la región y en la Argentina, trabajos que han estudiado específicamente la relación entre esos factores y la política exterior, buscando incluso relacionar las características específicas de la inserción internacional del continente. Sin embargo, creemos que es necesario revisar dicha literatura desde una determinada teoría del estado (Poulantzas, 1979), una perspectiva de sistema mundo, crítica $^{4}$ (Wallerstein, 1979; Cox,1981), que permita inscribir el análisis de la condición doméstica de la política exterior en relaciones sociales más amplias, en un enfoque histórico y de "tendencias profundas" (Renouvin y Duroselle, 2010). Solamente un abordaje histórico permite el análisis total y dinámico, abriendo paso a categorías explicativas válidas. Todos los aportes teóricos que aquí se realizan son fruto de investigaciones históricas, originados en preguntas constantes a diversas fuentes, como fondos de archivos desclasificados, entrevistas y corpus periodísticos, entre otras, porque tal como afirma Pierre Vilar, "no es legítimo creer que se ha dicho lo suficiente sobre un tema antes de haber confrontado la línea de reflexión elegida con un análisis profundo de las realidades, complejas en el espacio y cambiantes en el tiempo" (1980: p.8).

Así, todas las dimensiones de análisis que se desagregarán, deben ser siempre interpretadas desde una perspectiva que introduce la noción del factor tiempo, de la historicidad de los acontecimientos. Consideramos que los estudios en ciencias sociales deben incluir necesariamente el análisis de los distintos tiempos históricos, como los de flujo y los de creación o ruptura, los de larga duración y los cíclicos. Recupero aquí la visión de Vilar, y su discusión con Louis Althusser (Vilar, 1983) y Nicos Poulantzas respecto de las categorías teóricas, y la necesidad de remitirlas constantemente al estudio de los casos históricos concretos (Vilar, 1980) ${ }^{5}$; así como la importancia de comprender a los fenómenos de la política exterior como coyunturas, es decir, el "conjunto de condiciones articuladas entre sí que caracterizan un momento en el movimiento global de la materia histórica" (Vilar, 1980: p. 81). En esta línea, este trabajo tiene los siguientes objetivos que lo guían:

En primer lugar, recorrer brevemente las escuelas de pensamiento que han abordado la problemática de los condicionantes internos de la política exterior. Luego, desagregar las

\footnotetext{
${ }^{2}$ Pueden contarse como pioneras las obras de Wallerstein (1979), Strange (1988), Omahe (1990), Reich (1993), Rosenau (1997), Badie y Smouts (1995).

${ }^{3} \mathrm{La}$ inserción internacional es un concepto que remite al modo en que una nación se vincula con el resto de los actores y unidades del sistema mundial y se refiere por lo tanto a todo un conjunto de acciones establecidas a través de la política exterior y de la política interna. Incluye a su vez los comportamientos de diversos actores y sectores de la sociedad civil, es decir, por fuera del Estado. Coincidiendo con Perina (1988) entendemos por patrón de inserción cierta regularidad en la relación de una nación con el resto de los actores del sistema internacional. Es resultado de las diferentes políticas exteriores de los estados, pero también de las acciones internacionales de actores no-gubernamentales (p. 13). El estado tiene aún un rol relevante en la adopción y promoción de determinado patrón de inserción en el sistema mundo a través de la implementación de la política exterior y de la política económica interna y externa.

${ }^{4}$ La noción de teoría crítica es tributaria de conceptualizaciones elaboradas en el marco de la teoría crítica sociológica de la llamada escuela de Frankfurt, el núcleo de pensadores vinculados al Instituto de Frankfurt de Investigación Social establecido en 1923, entre cuyos miembros se destacan Max Horkheimer, Theodor Adorno, Herbert Marcuse y George Lukács, entre otros.

${ }^{5}$ Vilar insistía en la necesidad de distinguir entre formación económico-social real y el objeto teórico modo de producción, así como en la elaboración de métodos que permitieran analizar los casos concretos en que se combinan varios modos de producción, y evitar la aplicación de "modelos" al estudio histórico (1980).
} 
dimensiones de análisis para interpretar los condicionantes internos, pero a la luz de una clara teoría del estado, de una corriente crítica y de un enfoque histórico de las relaciones internacionales. En tercer término, es intención de este estudio complejizar la distinción entre factores internos y externos, teniendo en cuenta distintos niveles de análisis donde ambos se encuentran profundamente relacionados.

La mirada que recorre este artículo reivindica la noción de historia total, de Pierre Vilar (1980: 43-44), es decir una compleja trama de fenómenos que conforman la dinámica histórica y cuya comprensión implica una jerarquización analítica en base a la búsqueda de determinación, al mismo tiempo capaz de enlazar los diversos niveles de la actividad social y articular estructuras y acontecimientos. La historia total reconoce la preponderancia de los factores materiales en el devenir del proceso histórico, pero sin reducirlos a una determinación mecánica. Busca desentrañar sus vínculos con aspectos sociales, económicos, mentales, políticos y culturales. Implica a su vez comprender a las políticas exteriores como signo - desde una mirada sincrónica- como consecuencia o producto de modificaciones sociales pasadas, y como causa o factor de nuevas transformaciones futuras (Vilar, 1983: p.118).

\section{Los Análisis de Política Exterior (APE)}

La relación entre factores internos y externos de la política exterior ha sido abordada por la disciplina de las RI desde la década de 1950. En ese contexto se conformó un área de estudios denominada Análisis de la Política Exterior (APE). Es un campo que se ubica a medio camino entre las relaciones internacionales y la política comparada. Incluyó enfoques que buscaron construir modelos para interpretar el comportamiento del Estado y la formulación de su política exterior. El APE considera los factores que influyen en quienes toman la decisión e incluso en la decisión transformada en política, por eso profundiza sobre los factores internos de la política exterior. En sus inicios, estos abordajes se erigieron como respuesta a las tradiciones corrientes realistas, alrededor de un principal aporte: evitar la visión monolítica del Estado como actor racional unitario. En consecuencia, aparecieron en escena múltiples actores, intereses, grupos, que afectaban tanto la formulación como la implementación de la política exterior de un país. Surgieron casi exclusivamente en los Estados Unidos, y bajo la impronta del individualismo metodológico. Tuvieron la virtud de proponerse como teorías intermedias explicativas, incluir los aportes de otras ciencias sociales -como la sociología, la psicología y la psicología social- y buscar así explicaciones multicausales no solamente de la implementación de la política exterior sino también del proceso de formulación (Hudson y Vore, 1995).

Algunas de estas corrientes, en su afán de enfrentarse a las interpretaciones sistémicas de la política internacional, ponderaron de tal modo el comportamiento subjetivo de los actores en la formulación de la política exterior que los transformaron en equivalentes a los intereses del Estado. El modelo sistémico clásico de interpretación de la política exterior fue el de Kenneth Waltz (1979) ${ }^{6}$, y en efecto no era lo suficientemente útil para explicar en detalle la gestación de la política exterior; pero el reduccionismo subjetivista llevó a la exagerada ponderación de las percepciones de funcionarios, sin ningún tipo de explicación de carácter socioeconómico , o estructural (Hazleton, 1988:p. 13 y 14). Posteriormente, y abrevando en estas corrientes, aparecieron modelos más completos para comprender la política exterior: los de Graham Allison (1971) y el de Robert Putnam (1988) fueron quizás los más elaborados ${ }^{7}$.

\footnotetext{
${ }^{6}$ Los postulados de Waltz fueron derivando hacia la formulación del neorrealismo como corriente de las RI. Según sus postulados, el comportamiento de las unidades del sistema (estados) se explica mayoritariamente por constreñimientos estructurales del sistema que en los atributos o características de cada una de ellas (Waltz: 1979: 88-97).

${ }^{7}$ El enfoque de Robert Putnam (1988) fue denominado "de doble nivel". Tiene la gran virtud de mostrar el carácter difuso de la frontera entro lo interno y lo externo. En línea similar se expresaron los trabajos de Volgy y Schwarz (1991), señalando que muchas actividades gubernamentales tienen cada vez más una
} 
En nuestra región, los estudios sobre Política Exterior han sido los que más se han desarrollado dentro del campo de las RI, prevaleciendo de manera significativa sobre otras áreas de la disciplina (Deciancio, 2016). Dos autores han sido fundamentales y pioneros en el área: Alberto Van Klaveren $(1984 ; 1992)$ y Marcelo Lasagna (1996) ${ }^{8}$. El primero de ellos elaboró un modelo que incluyó la importancia de factores internos y externos en la definición de la política exterior. Realizó una operacionalización de unos y otros para lograr un esquema completo de interpretación de una decisión de política internacional. El objetivo fue poder superar una de las limitaciones centrales de la Teoría de la Dependencia, porque la cadena explicativa que construye sería para él demasiado abstracta como para permitir hipótesis sobre la interpretación de los procesos políticos y de la política exterior (Van Klaveren, 1984). Dos trabajos teóricos de Lasagna $(1995 ; 1996)$ se centraron sobre lo que el autor consideró como "determinantes interno s de la política exterior". Lasagna se dedica especialmente a la variable régimen político para explicar el comportamiento diferencial de los Estados frente a similares determinaciones del contexto externo. Sin embargo, hay en este enfoque reminiscencias de teorías sistémicas, carece de una teoría crítica que vincule esas variables con las relaciones sociales y los conflictos en el nivel del Estado, así como de un enfoque histórico. Volvemos a reiterar aquí que las categorías deben ser resultado del estudio de los casos históricos, y no pretender "encajar" los sucesos históricos en tipos ideales ${ }^{9}$.

En cuanto a la Argentina, a partir de mediados de los años noventa, dos aportes han cobrado mayor espacio en los estudios internacionales: las teorías constructivistas -a la $\mathrm{s}$ que haremos referencia-y los modelos que intentan explicar la variedad de conflictos intra-estatales en la formulación de la política pública. La $\mathrm{s}$ primera $\mathrm{s}$ se orientan a las cosmovisiones $\mathrm{o}$ creencias de los hacedores de política exterior, revalorizando el peso de las "ideas" y la "visión de mundo" en la adopción de determinada decisión en el área de la política internacional. La excelente obra de Paradiso (1994) tiene la virtud de historizar las trayectorias y debates en la política exterior argentina, vinculando las cosmovisiones con las lógicas del sistema político, las necesidades de la propia política económica nacional y el modelo de inserción internacional. Se rescata también el trabajo de Roberto Russell (1990), también pionero en la comprensión de las variables domésticas y en especial de las "creencias" en el análisis del curso de acción política ${ }^{10}$, así como el enfoque aplicado por Miryam Colacrai (2006), quien profundiza esta perspectiva de análisis.

Una serie de estudios e investigaciones desarrolladas bajo la dirección de Anabella Busso (2016) también han avanzado en la importancia de los factores domésticos en la política internacional, incluyendo múltiples variables y actores de las relaciones internacionales. Los resultados de proyectos de investigación colectiva reciente dan cuenta de un enfoque teórico y

dimensión externa. Sin embargo, el enfoque de doble nivel continúa explicando el accionar del Estado a partir de la maximización de beneficios, que pretenden equilibrar acontecimientos internos y externos. Profundizando esquemas interpretativos multinivel encontramos la obra de Kegley y Wittkopf (1995) que señalaron tres niveles analíticos: el individual, el nacional donde se aborda la política exterior de los Estados y sus condicionantes, y el nivel sistémico. Asimismo, entre los aportes al campo del APE, se destaca el análisis de las tres dimensiones de Carlsnaer: estructural, posicional e intencional (1992); y el trabajo de Hill, que profundiza la importancia de la vinculación dinámica entre actores y estructuras (2003). Un recorrido completo sobre los análisis de política exterior puede verse en (Llenderrozas y Finkielsztoyn, 2013)

${ }^{8}$ Se destacan también los estudios de Maira (1988) y Osorno (1995).

${ }^{9}$ En un trabajo anterior, se han desarrollado las falencias en ese sentido del concepto de autonomía heterodoxa de Juan Carlos Puig. Ver (Míguez, 2018).

10 Russell, considera que la estructura decisoria está integrada por los actores gubernamentales domésticos, los gobiernos extranjeros y los grupos no gubernamentales que tienen influencia sobre ella, determinando su tamaño, diferenciación de tareas y especialización, y a su vez diferenciándola de los procesos decisorios, como una dinámica de la evolución de la información, su alteración, y adopción de las decisiones. (Russell, 1990) 
metodológico ampliamente explorado ${ }^{11}$. Juan Gabriel Tokatlian y Federico Merke (2014), por su parte, han desarrollado la relevancia de los asuntos internos y de los agentes estatales en la formulación de las relaciones exteriores. En especial Merke se ha especializado en el estudio de las variables internas que explican cambios y continuidades en los procesos, y más recientemente (2019) ponderando las "preferencias" de los gobiernos en línea con los trabajos de Rhodes (2018). Este tipo de trabajos prácticamente abandonan la inscripción de esos comportamientos políticos e incluso individuales en procesos materiales e históricos. Las corrientes de la Economía Política Internacional (EPI) en la Argentina, desarrolladas especialmente en el equipo de la Facultad Latinoamericana de Ciencias Sociales dirigido por Diana Tussie, han abordado el estudio de actores diversos de la economía para comprender las relaciones internacionales, combinando la influencia de actores internos y externos.

Este trabajo parte de toda una línea de trabajo desarrollada por Mario Rapoport, similar a la explorada por Amado Cervo y la Escuela de Brasilia, que fueron quienes primero llamaron la atención entre la vinculación entre los modelos económicos y la política exterior, pero recuperando un enfoque histórico (Bernal-Meza, 2005) (Rapoport y Spiguel, 2006) ${ }^{12}$. Desde sus primeras obras, Rapoport (1982) desarrolló una particular mirada que vinculaba las variables económicas, sociales y políticas con las relaciones internacionales. Estos autores adoptaron una perspectiva metodológica inspirada en las tradiciones francesas de la Historia de las Relaciones Internacionales -las obras de Jean-Baptiste Duroselle y de Pierre Renouvin- un enfoque histórico-estructural del sistema-mundo que tiene origen en los trabajos de Emmanuel Wallerstein, y la ya citada perspectiva histórica de vinculació $\mathrm{n}$ entre estructura y coyuntura desarrollada por Vilar (1980). Tributando a esta corriente, continúa siendo necesario realizar un aporte conceptual al Análisis de la Política Exterior, que parta de una perspectiva crítica sobre el Estado, que atienda la situación de dependencia vigente de la región latinoamericana, y que al mismo tiempo pueda interpretar el accionar de los agentes individuales en el proceso de toma de decisiones de la política exterior, en el marco de tendencias económicas, sociales, culturales e ideológicas.

El ejercicio conceptual que sigue a continuación es fruto de variadas investigaciones históricas de las relaciones internacionales y resultado de una serie constantes de preguntas a los documentos, archivos, protagonistas, sucesos históricos donde puede comprobarse tanto la influencia de distintos factores domésticos, como el carácter complejo de dichas influencias (Míguez, 2013, 2015, 2016, 2017, 2019).

\section{Los condicionantes domésticos de la política exterior}

¿Cuáles son, entonces, esos condicionantes domésticos? Haremos un desagregado de las distintas dimensiones de análisis internas que consideramos pueden influir en la política exterior, haciendo las salvedades que se han afirmado desde inicio respecto de una distinción tajante con aspectos externos:

\section{a La composición del bloque en el poder, y por lo tanto, el estado de las relaciones de fuerza entre las fracciones que lo conforman y para con las clases subalternas.}

\footnotetext{
${ }^{11}$ Ver especialmente los trabajos de Busso A. (2016) y (2019), de Actis, E., Lorenzini, M. E., Zelicovich, J. (2016).

12 Simonoff (2012) describe a esta corriente como sociohistórica, e incluye a esta autora entre sus partícipes. Se destacan asimismo los trabajos de Rapoport y Madrid (2011), Morgenfeld (2011) y Kan (2015).
} 
La experiencia de la globalización neoliberal demostró que, lejos de perder poder, es el Estado quien se vuelve protector -y también garante- de los efectos de dicho proceso de mundialización del capital sobre la sociedad. Así, continúa siendo el momento de condensación de pujas en el espacio doméstico que impactan en la toma de decisiones. Este tipo de enfoque vuelve la mirada sobre el Estado, pero desde un punto de vista muy distinto al de los enfoques realistas o neorrealistas (Berringer, 2014). ¿Qué significa entonces, en esta perspectiva, analizar los condicionantes internos?

En primer lugar, hacer una interpretación de la relación entre Estado y sociedad (O’Donnell, 1978) para comprender el rol de las distintas clases y fracciones de clase en la formulación de la política pública. Un estado que es co-constitutivo de las relaciones económico-sociales, y que no puede ser comprendido sin atender esa profunda relación de la que emanan las instituciones, las políticas y las contradicciones (Hirsch, 2005) ${ }^{13}$. Afirmar que la política exterior es una política pública no constituye ninguna novedad. El tema es definir cómo interpretamos el Estado y su expresión en forma de políticas. Eso nos lleva a la necesidad de interpretarlas como parte de lo que Oscar Oszlak y Guillermo O'Donnell (1982) llamaron el Estado "en movimiento". La particularidad es que en el caso de las políticas exteriores, los sectores que instalan determinada cuestión como demanda no solamente se ubican en el plano interno, sino que se incluyen otros actores del plano internacional. Por tanto, la política es -al igual que las restantes- el resultado de un proceso social más abarcativo, que incluye a una multiplicidad de actores sociales, tales como clases, fracciones de clase, movimientos sociales, organizaciones e individuos estratégicamente ubicados respecto a una cuestión, además de los aparatos del Estado, todos los cuales toman posición respecto de ella (Thwaites Rey, 2005: p. 30-31). A esos actores se agregan los que pertenecen al sistema internacional, como las potencias, las empresas extranjeras, los organismos internacionales, etc. Todas esas disputas se condensan en el seno del Estado.

Es necesario identificar las fracciones de las clases dominantes que conforman el bloque en el poder, y cómo se configura o no la hegemonía en determinada coyuntura. Eso implica detectar los distintos actores económicos tanto en el plano de la sociedad civil como en su participación en el Estado, que pujan por determinada política internacional, y se expresan a partir de sus prácticas económicas directas, su organización en corporaciones, y ejerciendo una influencia indirecta en los ámbitos estatales. La hegemonía de una de esas fracciones de clase es significativa a la hora de interpretar la estrategia de política exterior de determinado gobierno. Asimismo, las presiones o negociaciones con otros sectores relativamente desplazados también impactan en las decisiones internacionales, sea por exclusión de sus demandas, o por determinada incorporación particular que permita "balancear" el poder.

En las condiciones de un capitalismo globalizado, la inserción en dicho escenario mundial es un elemento central para definir las posibilidades o los límites de cualquier proyecto económico, político y social nacional. En el caso de países periféricos, en los períodos donde las fracciones que hegemonizan tienen estrechos vínculos de asociación con potencias extranjeras, con intereses de corporaciones transnacionales, o bien su supervivencia está atada casi exclusivamente a la relación con actores del sistema global, la política exterior se constituye en un instrumento de acercamiento o de ratificación de la predominancia de dichos vínculos. Ello puede evidenciarse a lo largo de nuestra historia reciente en numerosas ocasiones: tratados comerciales, privilegios otorgados en concesiones, procesos de endeudamiento fraudulentos, licitaciones irregulares, legislaciones hechas "a medida" de determinada empresa o corporación, entre otros ${ }^{14}$.

\footnotetext{
${ }^{13}$ O’Donnell afirma que "la garantía que presta el Estado a ciertas relaciones sociales, incluso las relaciones de producción que son el corazón de una sociedad capitalista y de su contradictoria articulación en clases sociales, no es una garantía externa ni posterior de dicha relación. Es parte intrínseca y constitutiva la misma, tanto como otros elementos económicos, de información y control ideológico que son aspectos que sólo podemos distinguir analíticamente en dicha relación" (1978: 1162).

${ }^{14}$ Para un análisis de las relaciones internacionales de la periferia, desde la perspectiva de la regulación, ver (Mistral, 1978)
} 
Las corrientes de la EPI focalizan en esquemas de ganadores y perdedores, para comprender las relaciones de poder donde tienen anclaje las políticas internacionales. Por esa razón, aparecen actores económicos como las empresas, a las que consideran como actores políticos "no solo porque pueden interferir en las prerrogativas del Estado (marcando su agenda, con anuncios de inversión o desinversión), sino porque se relacionan de modo permanente con el Estado y sus diferentes estamentos" (Tussie, 2015: p.160). Al mismo tiempo, hay que tener en cuenta que los intereses vinculados a la asociación con las potencias hegemónicas y que -por lo tanto- garantizan la dependencia, se presentan disputando dentro del bloque en el poder y no como una constricción "externa" solamente. En condiciones de dependencia, esa disputa dentro del bloque de poder incluye las relaciones de asociación subordinada respecto de las potencias dominantes del sistema internacional, y a su vez, las pujas entre las potencias por el predominio en cada uno de los países en cuestión (Rapoport, 1982; Morgenfeld, 2011, Laufer 2008).

\section{b El grado de autonomía relativa del estado, la coyuntura política y del sistema político, y el nivel de organización de los sectores subalternos respecto de cuestiones socialmente problematizadas vinculadas con la política internacional.}

En cada período histórico, las dirigencias políticas expresan tanto las disputas de las distintas fracciones dominantes como las demandas provenientes de las amplias mayorías populares, y ello merece ser evaluado en forma particular para comprender la política exterior. Los grados de autonomía relativa del Estado (Poulantzas, 1976) tienen impacto en la formulación y en la implementación de esta política. Este aspecto tiene relación con las características del régimen político, con las condiciones particulares del funcionamiento del sistema político y con la condición de legitimidad de los gobernantes (Luján, 1993). En las democracias electorales cobra relevancia la escena política y el rol de los partidos , 1 as corrientes ideológicas que expresan, sus propuestas, la dinámica del sistema electoral, el funcionamiento de los poderes de estado, y en especial del Poder Legislativo (Sánchez; 2013; López Burian, 2015) ${ }^{15}$.

El conflicto es un elemento constante tanto entre las fracciones de clase como respecto de los sectores subalternos, lo que explica supuestos "vaivenes" en la política internacional. En la política exterior también podemos leer un determinado estadío de las relaciones sociales de fuerza. Tal como afirma Musacchio (2016) la conflictividad social no desaparece cuando los sectores dominantes logran imponer sus intereses económicos desde el plano político, como tampoco desaparece la tensión sobre la forma en que el país debe articularse con el sistema internacional (p. 34).Por lo tanto, la política exterior, al igual que otras políticas públicas, también expresa el modo en el que se condensan los intereses de los distintos sectores sociales, incluidos los subalternos. En el seno mismo del Estado se expresa esa conflictividad, que en algunos momentos abre espacio a la ampliación de lo político, permitiendo el avance de demandas y cuestiones socialmente problematizadas provenientes de sectores no hegemónicos 16.

\footnotetext{
${ }^{15}$ La tesis doctoral de Leandro Sánchez (2013) desarrolla el rol del Parlamento argentino en la elaboración de la política exterior argentina, desde una perspectiva de los condicionantes internos. Sánchez construye "una concepción de política exterior entre el proceso político interno y externo donde dicha distinción por una u otra posición no constituye una elección teórico-filosófica irreversible, sino como una cuestión de orden empírico donde el peso de cada una de ellas como factor explicativo de la política exterior se ajusta a hechos concretos" (2013: 34).

${ }^{16}$ René Zavaleta Mercado, abocado a la comprensión de la problemática de los países periféricos, ha considerado que de acuerdo a las fases históricas pueden encontrarse en los casos específicos, situaciones instrumentales del Estado y fases de autonomía relativa (2009: 332). El manejo del Estado en forma directa por parte de las clases dominantes, sin mediación, genera una reducción de margen de lo político.
} 
La experiencia latinoamericana muestra que bajo ciertas circunstancias, se torna factible que desde el propio Estado se desplieguen mecanismos de resistencia a los aspectos más perversos del capital para la vida de los pueblos, poniéndose de manifiesto la dimensión contradictoria del Estado. En concreto, cuando las relaciones de fuerzas permiten que en los Estados se articulen procesos políticos y sociales, nacionales y regionales, impulsados por movimientos populares, se abre la posibilidad de empujar políticas favorables a ciertas demandas e intereses de las clases y grupos subalternos (Thwaites Rey y Ouviña, 2016) ${ }^{17}$. Ya en su obra Todo imperio perecerá, Duroselle (1992) analiza aspectos internos que tienen impacto en la política exterior. Entre ellos destaca: la presión del parlamento -que a su vez se relaciona con la influencia de formaciones políticas y de grupos de interés-; la lectura que los responsables de la política hacen del "ambiente" -ideas predominantes, opinión pública, climas sociales; y lo que denomina "estrategia de la derivación", que refiere a los casos en los que un gobierno utiliza la política exterior para modificar posiciones respecto de la política interna o para debilitar a la oposición. Quiere decir que es necesario continuar teniendo en cuenta el impacto que tienen en la política exterior lo que el propio Duroselle llamó "comunidades intermedias organizadas": partidos políticos, organizaciones de profesionales, asociaciones diversas de naturaleza política y cultural, así como el impacto de sus posiciones en los cuerpos electorales (Guillen, 2012). Un caso de estudio en los últimos años son las diversas corrientes y movimientos religiosos, su impacto en la política y por lo tanto, en la política internacional (Del Campo y Resina, 2020).

Es por esa razón que, en algunos contextos y coyunturas particulares, la movilización social y la presión de sectores subalternos son también variables a considerar en el proceso de toma de decisiones de la política exterior, como se evidencia en numerosos casos de la historia argentina (Míguez, 2012; Míguez y Morgenfeld, 2017) ${ }^{18}$.

\section{c El modelo económico o tipo de desarrollo nacional}

Definir un modelo económico, una estrategia o las características del desarrollo de una nación implica analizar tanto condiciones estructurales históricas de mediano y largo plazo, como los impactos concretos y coyunturales de las políticas económicas adoptadas por los sucesivos gobiernos. Dicho modelo condiciona el tipo de política exterior. Utilizamos aquí la noción de desarrollo en el sentido expresado por Aldo Ferrer (2007), quien afirma que "el desarrollo implica la organización e integración de la creatividad y los recursos de cada país para poner en marcha los procesos de acumulación en sentido amplio" (p. 432). Por eso se ha decidido referirse en la categorización a modelos económicos, porque no todos ellos implican estrategias de desarrollo ${ }^{19}$. Se retoma también el concepto de régimen o patrón de acumulación utilizado por Basualdo que "alude a la articulación de un determinado funcionamiento de las variables económicas, vinculado a una definida estructura económica, una peculiar forma de Estado y las luchas entre los bloques sociales existentes" (2007: p. 6), porque permite vincular las variables económicas con lo que hemos desarrollado en los puntos 1 y 2.

\footnotetext{
${ }^{17}$ De acuerdo con los autores, a costa del efecto pasivizador de ciertos beneficios otorgados por políticas estatales (2016).

${ }^{18}$ Ver el trabajo de Leandro Sánchez y la introducción en este volumen.

19 Ferrer afirma: "Un país puede crecer, aumentar la producción, el empleo y productividad de los factores, impulsado por agentes exógenos, como sucedió con Argentina en la etapa de la economía primaria exportadora. Pero puede crecer sin desarrollo, es decir, sin crear una organización de la economía y la sociedad capaz de movilizar los procesos de acumulación inherentes al desarrollo o, dicho de otro modo, sin incorporar los conocimientos científicos y sus aplicaciones tecnológicas en el conjunto de su actividad económica y social" (2007: 433)
} 
Lo que se entiende aquí como modelo económico -aspectos analíticamente distinguibles como económicos dentro del patrón de acumulación- puede desagregarse en una serie de variables que lo componen:

A) la composición de la estructura productiva (desarrollo de las distintas ramas productivas, crecimiento, integración, diversificación, concentración, participación del capital extranjero, y el tipo de relación entre Estado y Mercado)

B) las características del denominado sector externo, incluyendo tanto el comercio exterior y el tipo de política aplicada (el tipo de inserción comercial global, composición y destino de las exportaciones e importaciones, relación con organismos multilaterales específicos) como la cuenta de servicios, los movimientos financieros y de bienes de capital internacionales, y los pagos a las instituciones internacionales (relación con FMI, BM y otras entidades similares)

C) la utilización del crédito público, privado y el internacional

D) la distribución del ingreso y la participación de los distintos sectores sociales en el producto total, las políticas de empleo, sociales, educativas y en el área de salud (aquí se incluyen elementos más amplios como la absorción de mano de obra y la formalidad del mercado laboral).

E) la orientación del desarrollo científico y tecnológico

Este tipo de enfoque evita caer en las interpretaciones "comercialistas", que reducen la relación entre economía y política internacional al aspecto comercial, sin tener en cuenta todas las áreas en las que las economías nacionales e internacional están vinculadas ${ }^{20}$.

En cuanto a la primera de las variables, es central identificar como condicionante de la política exterior, qué tipo de actividades económicas caracterizan la estructura productiva, el grado de concentración $\mathrm{y}$ fundamentalmente cuáles son promovidas, beneficiadas $\mathrm{o}$ perjudicadas por acción u omisión de la política pública. Es un elemento crucial porque dichos intereses también tendrán expresión en el seno del estado y en la formulación de la política exterior de acuerdo a la gravitación que tengan en la estructura productiva.

La participación del capital extranjero así como sus condiciones de operación en el plano local, al igual que la incorporación a cadenas productivas transnacionales, también son un elemento a ponderar, tanto porque implican tener en cuenta la relación con esos capitales - y de múltiples actores- a la hora de la toma de decisiones, como por las lógicas de intermediación que se generan en el ámbito productivo (Musacchio, 2020).

La relación que se establezca entre el Estado y el mercado, apunta a incluir como elemento condicionante el tipo de políticas que orientan la intervención política en el mercado a través de reglas de juego, regulaciones y participación en distintos ámbitos de la esfera económica. Como afirma Ferrer (2007) el orden global proporciona un marco de referencia para el desarrollo de cada país, "pero la forma de inserción en su contexto externo depen de, en primer lugar, de factores endógenos, propios de la realidad interna del mismo país" (p. 434).

Las variable $\mathrm{B}$ tiene impacto en una problemática que ha sido central en el caso de los países periféricos y que es la restricción externa, que se ha convertido en un condicionante recurrente al crecimiento económico. Existe una amplia literatura en la historia económica que ha abordado esta problemática no solamente desde sus aspectos coyunturales sino también estructurales Cuestiones como los flujos de capitales asociados a pagos de intereses, remisión de utilidades y dividendos, inversiones extranjeras y fugas de capitales son centrales a la hora de definir la estrategia económica y cómo impacta en los diversos actores. Por otra parte, es el aspecto donde existe una total imbricación entre política interna y externa, ya que todas las

\footnotetext{
${ }^{20}$ Sí existe más literatura respecto de la relación entre factores domésticos y externos en el ámbito de las negociaciones comerciales. Ver (Conceicao-Heldt, 2013) y (Betz, 2015)
} 
medidas están estrechamente vinculadas con actores del sistema internacional, sean unidades nacionales, organismos, empresas. Entre los trabajos que han desarrollado más claramente la relación entre los proyectos económicos y la política internacional se destacan los de Rapoport (2020), los de Moniz Bandeira (2006) (2013), Cervo (2003), Vidigal (2009), Musacchio (2020) (2016), Brenta (2019) Laufer (2008).

El endeudamiento externo se ha transformado en un gran condicionante de la política exterior, y es parte central en la definición del modelo económico (Brenta, 2019). Organismos multilaterales como el Fondo Monetario Internacional se han constituido en actores políticos que penetran las fronteras del Estado de modos diversos y condicionan las políticas públicas adoptadas en áreas no solamente económicas sino también sociales, culturales e incluso de seguridad interna.

\section{d La formación intelectual y profesional de los hacedores de política, así como las ideas y creencias que guían la toma de decisiones.}

Las trayectorias individuales y de actores colectivos son relevantes a la hora de interpretar la toma de decisiones en el proceso de formulación de la política exterior y en su implementación. En algunas circunstancias es una variable central para comprender diferencias, vaivenes, contradicciones (Rapoport, 2016). Las perspectivas constructivistas han hecho un gran aporte que destaca la importancia de las cosmovisiones. Alexander Wendt (1995) sostuvo que las asociaciones humanas se encuentran esencialmente determinadas por las "ideas compartidas". Plantea que las estructuras sociales poseen tres características: el conocimiento compartido -o entendimiento intersubjetivo-, los recursos materiales y las prácticas (Wendt, 1995: p.73-74). Este tipo de análisis buscaba en esa clave interpretar tendencias hacia la cooperación o hacia el conflicto.

Gran parte de los trabajos que adscriben a esta perspectiva se han orientado a los estudios sobre las organizaciones internacionales, la construcción de normas, o comportamientos sociales de oficinas públicas y su impacto en las negociaciones internacionales. Al mismo tiempo han abordado la existencia de comunidades epistémicas y su rol en la construcción de identidades, intereses y tipos de organización social. Según la definición de Peter Haas (1992), una comunidad epistémica es una red de profesionales con reconocida experiencia y competencia en un campo determinado y un reconocido conocimiento de temas relevantes para la elaboración de política en ese terreno o área temática. El constructivista Emanuel Adler (1992) concibe a las comunidades epistémicas como creadoras de creencias intersubjetivas que actúan como "vehículos de supuestos teóricos, interpretaciones y significados colectivos que pueden ayudar a crear la realidad social de las relaciones internacionales" (p. 343). En una deriva más reciente, Adler y Pouliot (2011) incorporan el concepto de prácticas internacionales, para el análisis de los comportamientos y dinámicas globales, considerando al mundo "no como un conjunto de estados ni dividido por fronteras y líneas de identificación nacional, sino como comunidades transnacionales de práctica" (p. 29).

Tal como afirma Robert Frank (2012), los historiadores de las relaciones internacionales han sido constructivistas sin saberlo, antes de que el constructivismo fuera teorizado, ya que la noción de "fuerzas profundas" los obligó a medir el peso de las mentalidades, estereotipos e imaginarios sociales que conforman la percepción de las realidades (p. 72).

Estas instituciones, ideas y valores no flotan en el espacio, sino que están ancladas (aunque no en forma lineal) a procesos materiales, es decir, al devenir histórico de la manera en que se estructura el escenario nacional. "Toda investigación sobre las representaciones no debe olvidar las duras realidades (...) Deconstruir lo real no significa negarlo" (Frank, 2012: p.75).

En este sentido, es importante comprender esas trayectorias individuales en corrientes de pensamiento, circulación de conocimiento, instituciones donde se realizan los recorridos formativos, los debates ideológicos del período en el que piensan y actúan. Y relacionarlas con condiciones materiales e ideacionales de mediano y largo plazo. Existe una dimensión 
ideológica de toda diplomacia. Y esa dimensión no está siempre claramente definida en postulados duros y coherentes, sino que su fuerza suele residir en sentimientos periféricos que ellas generan incluso en espacios que no comulgan directamente con esas ideologías (Frank, 2012: p. 353). Los imaginarios y las prácticas culturales que tienen influencia en la definición de la política exterior responden, en algunos casos, a las fuerzas profundas descriptas por Renouvin y Duroselle (2010): las "mentalidades colectivas" o "subconscientes", como por ejemplo en el caso de los nacionalismos, las tendencias neutralistas o pacifistas de algunas naciones, imágenes sociales y culturales, percepciones geopolíticas, que pueden constituirse en terreno favorable para una u otra decisión política (p. 243- 245) ${ }^{21}$.

Ello nos lleva a la reflexión de por qué y en qué condiciones las élites dominantes y las dirigencias políticas de los países dependientes adoptan o no los paradigmas hegemónicos de las potencias dominantes para la formulación de sus políticas públicas (Míguez, 2013). El traspaso del conocimiento desde las potencias a los países dependientes constituye uno de los elementos fundamentales para la preservación de un orden internacional jerárquico, puesto que a través de universidades y centros de difusión de conocimiento se construye la hegemonía, el aspecto consensual de la dominación (Ramirez, 2007). La transmisión de paradigmas mediante la enseñanza en las escuelas diplomáticas, academias militares, universidades, etc, contribuye a sostener la legitimidad de dicho orden y la existencia de una inserción internacional subordinada. También se acuerda con Arlene Tickner (2002) cuando afirma que el predominio de las vertientes dominantes de la ciencia social occidental en los países subdesarrollados tiene el efecto de negarles a éstos la condición de sujetos activos en la construcción de su propio conocimiento y cualquier proceso de transmisión de conocimiento entre norte y sur está atravesado por la dinámica de la dominación, la explotación y la hegemonía. Es decir que, en términos metodológicos, es necesario reconstruir el entramado de ideas que sustentan la política exterior en el marco sistémico de las relaciones de poder en el mundo (Deciancio y Míguez, 2020). Asimismo, hay que evitar aquí las caracterizaciones lineales. Tal como ha desarrollado Corigliano (2008), los hacedores de política exterior, presidentes, cancilleres o funcionarios que siempre actúan respondiendo a sistemas de creencias y visiones teóricas explícitas o implícitas- no suelen actuar en el marco de paradigmas únicos, sino que recurren "a conceptos precedentes de diversos, y a menudo contradictorios, enfoques teóricos (p. 8).

Una vez realizado este análisis pormenorizado de los condicionantes internos de la política exterior, pasaremos a reflexionar sobre la vinculación entre los denominados factores internos y externos.

\section{Conclusiones ¿cuán domésticos son esos condicionantes?}

Abordar un análisis exhaustivo de las dimensiones de análisis que componen los factores condicionantes internos de la política exterior es útil fundamentalmente en varios sentidos: a) permite identificar en cada caso qué variable tiene mayor gravitación para explicar cambios o continuidades, vaivenes y contradicciones; b) habilita a realizar comparaciones de manera más clara, tanto entre distintos períodos en el caso de una misma nación, como entre políticas exteriores de distintos países; c) revaloriza al espacio nacional como ámbito donde surgen también las posibilidades de transformación política.

Sin embargo, tal como se ha anticipado desde la introducción, en cada una de las dimensiones de análisis descriptas en nuestro apartado anterior, podemos encontrar la influencia y relación con movimientos, actores, clases y flujos transnacionales. Esta problemática ha sido tratada en la literatura de las Relaciones Internacionales. Por ejemplo, el término "interméstico" - muy

\footnotetext{
${ }^{21}$ Duroselle (1974) diferencia las mentalidades colectivas, entendidas como actitudes mentales precisas nacionalismo, colonialismo, pacifismo, etc.- que se encuentran en una posición intermedia entre las ideologías -que serían más duraderas- y la opinión pública. Para una correcta definición de opinión pública en este sentido, ver (Frank, 2012: p.358).
} 
utilizado en la disciplina- fue acuñado por Bayless Manning en 1977, y se trata de una expresión compuesta que describe aquellos asuntos que son inseparablemente tanto internacionales como domésticos. El autor afirmaba así que "la naturaleza de los asuntos internacionales contemporáneos está marcada por ser simultánea, profunda e inseparablemente internacional cuanto doméstica" (Manning, 1977:p. 309). Desde una perspectiva marxista, Poulantzas (1976) también afirmaba que factores internos y externos no podían interpretarse en forma aislada. Especialmente desde el avance de la denominada globalización de fines del siglo XX, encontramos ideas de ese tipo en los enfoques de Rosenau y su noción de "nueva frontera" (Rosenau, 1997: p.4-5) y en el concepto de "espacio social transnacional" de Beck (1998). Rosenau (1997) sostuvo que los cambios relacionados con esa nueva condición de interdependencia global, promovieron formas intensas de vinculación global, incluyendo a los movimientos sociales.

Lo cierto es que la Historia de las Relaciones Internacionales tuvo siempre clara esa interconexión, puesto que cuando se estudian los fenómenos reales, las características o categorías estanco parecen ser inútiles. Durosselle afirmó que "no existe ningún acto de política extranjera que no tenga un aspecto de política interna (...) todo acto que mira hacia el extranjero tiene un aspecto interior, aunque más no sea en función del simple hecho de que la autoridad responsable emana de un proceso interno" (1992: p. 47). Llega a esa conclusión a partir de sus extensos estudios históricos que lo comprueban. Luego, a las corrientes de estudios transnacionales se sumaron las de historias conectadas y los Estudios Globales, que profundizaron la reflexión al respecto, dando origen a toda una serie de conceptos híbridos que buscan nombrar esa complejidad entre espacios locales y globales ${ }^{22}$. Las corrientes de la EPI también tienen como una de sus premisas la estrecha vinculación entre lo interno y lo externo. Tal como afirma Tussie, "la problemática de la EPI se define por la influencia recíproca de la economía en la política (y viceversa), así como de lo interno en lo externo (y viceversa)" (2015: p.161). Pero vayamos ahora al tratamiento de nuestras variables domésticas y su carácter transnacional, entrelazado, conectado con dinámicas regionales y mundiales.

En cuanto a la composición del bloque en el poder debemos incluir allí la injerencia de actores transnacionales que operan a través de sus vínculos con distintas clases y fracciones, dadas las particulares características de las formaciones económico-sociales dependientes. Berringer (2014) explica que los bloques de poder de los países dependientes se articulan en torno a la relación que las fracciones de capital endógeno establecen con el capital imperialista, y que las contradicciones entre las potencias predominantes del sistema internacional pueden influir tanto en nuevas configuraciones de poder en el seno del bloque dominante del país dependiente, como en el de dicha potencia (p. 447). Al mismo tiempo, los movimientos sociales también pueden constituirse en parte de un entramado transnacional, que incluso los transforma en ocasiones en sujetos globales. La presión de movimientos de trabajadores, de intelectuales, sociales, ambientalistas, feministas, etc. que actúan rebasando las fronteras estatales impacta en el plano local, y constituye además una fuente de demanda para los Estados nacionales y, por lo tanto, exige definiciones de política exterior por acción u omisión.

El modelo económico tipo de desarrollo está inserto en una estructura global, y tiene existencia en un entramado complejo donde se develan limitaciones y posibilidades en el marco de determinado proceso de acumulación mundial. Las variables operacionalizadas relacionadas con la estructura productiva, el comercio exterior, el sector externo, el endeudamiento, entre otras, incluyen la relación con las potencias predominantes del sistema internacional, organismos multilaterales y también con otros países del globo. Se toma aquí la perspectiva de Bob Jessop (2011), quien ha desarrollado extensamente la relación entre el Estado y el mercado global, afirmando que se trata de un vínculo complejo a partir de tres razones: la importancia tanto de las dimensiones territoriales como de flujo; la condición de los Estados de "conectores de poder" que funcionan como nodos en una red; y el hecho de que la integración en el mercado

\footnotetext{
${ }^{22}$ Para una reflexión sobre los Estudios Globales en las RRII ver el número 102, año 2020, de la Revista Colombia Internacional, Dossier compilado por Jean-Marie Chenou y Cinthia Quiliconi. Ver también Figallo y Henríquez (2020).
} 
mundial no solo condiciona a los gestores del Estado, sino también al capital y al trabajo (p. 2930). Por su parte, Cox (1981) afirma que la hegemonía a nivel internacional no es un orden entre Estados, sino un orden dentro de una economía mundial con un modelo de producción dominante que penetra en todos los Estados y los vincula a otros modelos de producción subordinados, a la vez que se erige como un complejo de relaciones sociales internacionales que conectan las clases sociales de los diferentes países. Asimismo, existen importantes estudios sobre la influencia de las empresas transnacionales y los organismos globales en la política interna de los países periféricos (y de los que no lo son) ${ }^{23}$.

Las identidades nacionales, la visión del otro, las mentalidades subjetivas y las imágenes culturales de los hacedores de política se construyen también por interacción de una matriz nacional y otra transnacional (Grosser, 2012: p. 283). La formación intelectual, así como las ideas y creencias, están profundamente relacionadas con la circulación de saberes, donde el flujo desde el centro a la periferia sigue siendo muy notorio y predominante. Se incluye, como lo hacía Van Klaveren (1992), el sistema de creencias como factor interno de la política exterior, pero en este caso volvemos a poner en tensión el carácter puramente doméstico. Incluso, tal como lo ha desarrollado la Historia de las Relaciones Internacionales, las concepciones del orden internacional se transforman en clivajes internos y se expresan en posiciones divergentes a la hora de la toma de decisiones (Guillen, 2012). Por todas estas razones es que para el Análisis de la Política Exterior hemos desarrollado en otro trabajo conjunto con Melisa Deciancio (Deciancio y Míguez, 2020) un esquema que incluye, más que factores internos y externos, cuatro niveles de análisis donde condicionantes internos y externos se encuentran profundamente relacionados:

a) Nivel global. Este nivel, más amplio, incluye tanto el sistema interestatal tradicionalmente objeto de estudio de las RI, como un nivel global, que comprende una multiplicidad de actores estatales y no estatales que rebasan las fronteras del Estado. En esta etapa del análisis, se evalúan los vínculos con las potencias predominantes y con los actores, instituciones y estructuras con capacidad de incidir manteniendo o disputando el orden global.

b) Nivel de los Estados pares. Las relaciones con Estados pares pueden pertenecer a un proceso de integración regional o simplemente referirse a socios cercanos cuyos vínculos son relativamente simétricos.

c) El Estado como relación social. Se parte de considerar al Estado como condensación de fuerzas para poder observar el modo en que allí se expresan las pujas dentro del bloque dominante, así como el vínculo entre Estado y sociedad civil, las relaciones de fuerza y la situación de los sectores subalternos, pero incluyendo también en las tramas políticas elementos, actores y situaciones que se refieren en reiterados casos a condicionantes de orden transnacional.

d) Actores e ideas. Este nivel tiene por unidad de análisis la dirigencia política, los funcionarios, los grupos de presión y los think tank, y remite a la interpretación del rol de las ideas y la circulación de conocimiento, tanto a nivel doméstico como aquellas determinadas por los flujos globales de ideas e información (Deciancio y Míguez, 2020: p. 97-98).

Como se puede observar, en estos niveles están incluidos los condicionantes internos, pero en relación con las influencias o condiciones externas. Este esquema no quita relevancia a la importancia de desagregar y distinguir la condición interna, pero es útil para hacer un análisis e interpretación completos de la política exterior, especialmente de los países que no cuentan entre las potencias del globo.

Como se ha afirmado en la introducción, este artículo es el resultado de varios años de investigación histórica y conceptual en el área de las Relaciones Internacionales, la Economía Política y la Ciencia Política. Resuenan en él los enfoques de colegas, puesto que todo conocimiento es en última instancia, un ejercicio colectivo. Desarrolla un particular abordaje que combina una perspectiva crítica del Estado y del sistema internacional, con un enfoque

\footnotetext{
${ }^{23}$ A este respecto ver (Amin 1974, 1988; Sunkel 1972)
} 
histórico y multidisciplinario. Ello nos permite elaborar una clasificación de las variables domésticas que consideramos necesarias a tener en cuenta a la hora de analizar el proceso de formulación e implementación de la política exterior. Así, distinguimos cuatro condicionantes centrales: uno relacionado con la composición del bloque en el poder, otro con la autonomía relativa del estado y la actuación de los sujetos políticos, en tercer lugar el modelo económico, y por último la cosmovisión y sistema de creencias de los hacedores de política.

Retomando los objetivos iniciales, se ha pretendido aportar a un debate actual en el campo de las RI, que debe incluir las miradas de zonas del mundo que no cuentan entre las potencias hegemónicas, y cuya genuina preocupación es la consecución de un orden justo que permita el bienestar de amplias mayorías. Fundamentalmente, se reivindica la posibilidad de profundizar las conceptualizaciones en la medida en que son resultado de los estudios de los fenómenos concretos, un ejercicio sin duda más complejo. La aplicación de tipologías abstractas a los estudios de caso constituye también un modo de imposición de conocimientos generados con determinados fines e intereses. La Teoría de las Relaciones Internacionales en las zonas periféricas del mundo requiere reivindicar más que nunca el enfoque histórico para poder pensarse a sí misma. Al mismo tiempo, revalorizar el espacio nacional como ámbito de construcción de alternativas es un desafío totalmente vigente. Por lo tanto, revisar las condiciones internas que posibilitan mejores estrategias de inserción internacional es una tarea necesaria de toda reflexión comprometida con un desarrollo económico, social, político y cultural justo.

\section{Referencias bibliográficas}

Actis, E., Lorenzini, M. E., Zelicovich, J. (2016). Modelo de desarrollo y estrategia de inserción: claves para la interpretación de su relación. En Busso, A. (Coord.), Modelos de desarrollo e inserción internacional: aportes para el análisis de la política exterior argentina desde la redemocratización 1983-2011 (pp. 15-30). Rosario, Argentina: Universidad Nacional de Rosario Editora.

Adler, E. (1992). The emergence of cooperation: National epistemic communities and the international evolution of the idea of nuclear arms control. International Organization, 46 (1), pp. 101-145.

Adler, E. y Pouliot, V. (2011). International practices. International Theory, 3 (1), pp. 1-36. Recuperado de: https://bit.ly/3j2EEj2.

Amin, S. (1974). Capitalismo periférico y comercio internacional. Buenos Aires, Argentina: Periferia.

Allison, G. (1971). Essence of Decision: Explaining the Cuban Missile Crisis. Boston, Estados Unidos: Little Brown.

Badie, B. y Smouts, M. (1995). Le retournement du monde. Sociologie de la scène internationale. Paris, Francia: Presses de Sciences-Po/ Dalloz.

Basualdo, E. (2007). Concepto de patrón o régimen de acumulación y conformación estructural de la economía. Maestría en Economía Política Argentina-FLACSO Argentina. Documento $\mathrm{N}^{\mathrm{o}}$ 1. Recuperado de: https://bit.ly/2Hi4ZLI.

Beck, U. (1998). ¿Qué es la globalización? Falacias del Globalismo, Respuestas a la Globalización. Barcelona, España: Paidos. 
Bernal-Meza, R. (2005). América Latina en el mundo. El pensamiento latinoamericano y la teoría de las relaciones internacionales. Buenos Aires: Nuevo Hacer-Grupo Editor Latinoamericano.

Berringer, T. (2014). Nicos Poulantzas e os estudos de relaçoes internacionais. Quaestio Iuris, 7 (2), pp. 433-452. Recuperado de https://bit.ly/364f3Cq.

Betz, T. (2015). Trading Interest: Domestic Institutions, International Negotiations, and the Politics of Trade. Michigan, Estados Unidos: University of Michigan. Recuperado de: https://bit.ly/361DMqY.

Brenta, N. (2019). Historia de la deuda externa de Martínez de Hoz a Macri. Buenos Aires, Argentina: Capital Intelectual.

Busso, A. (2016). Modelos de desarrollo e inserción internacional: aportes para el análisis de la política exterior argentina desde la redemocratización 1983-2011. Rosario, Argenitna: Universidad Nacional de Rosario Editora.

Busso, A. (2019). El vínculo entre los condicionantes internos y la política exterior. Reflexiones sobre el caso argentino. Ciclos en la Historia, la Economía y la Sociedad, 52 (2), pp 332 Recuperado de: https://bit.ly/2RSO3xy.

Carlsnaer, W. (1992) "The Agency-Structure Problem in Foreign Policy Analysis".International StudiesQuarterly, (36)3, pp. 245-270. Recuperado de https://bit.ly/2FWIkEm.

Cervo, A. (2013). Conceptos en relaciones internacionales. Relaciones Internacionales, 22, pp. 149-166. Recuperado de: https://bit.ly/3kFXOeF.

Colacrai, M. (2006). La marcha de la integración en América Latina. El rol de las ideas, instituciones y políticas en el Mercosur. En Boron, A. y Lechini, G. (Ed.), Política y movimientos sociales en un mundo hegemónico. Lecciones desde Africa, Asia y América Latina. (pp. 381-397). Buenos Aires, Argentina: Clacso. Recuperado de: https://bit.ly/2FZOgOh.

Conceicao-Heldt, E. (2013). Two-level games and trade cooperation: What do we now know?. International Politics, 50 (4), pp. 579-599.

Corigliano, F. (2008). Híbridos teóricos y su impacto en la política exterior: El caso de los gobiernos de Néstor y Cristina Kirchner. Boletín del Instituto de Seguridad y Asuntos Estratégicos, 47 (11), pp. 8-10.

Cox, R. W. (1981). Social Forces, States and World Orders: Beyond International Relations Theory. Millennium: Journal of International Studies, 10 (2), pp. 126-155. Recuperado de https://bit.ly/305bfx8.

Deciancio, M. (2016). La construcción del campo de las Relaciones Internacionales en Argentina. Buenos Aires, Argentina: FLACSO Argentina.

Deciancio, M. y Míguez, M. C. (2020). Contribuciones de los estudios globales al análisis de la política exterior: una aproximación metodológica. Colombia Internacional, 102, pp. 87112. Recuperado de: https://bit.ly/3kAKiZQ.

Del Campo, M. E. y Resina, J. (2020). ¿De movimientos religiosos a organizaciones políticas? La relevancia política del evangelismo en América Latina. Documentos de Trabajo (Fundación Carolina), 35.

Dos Santos, T. (2003). La teoría de la dependencia. Balance y perspectivas. Buenos Aires, Argentina: Plaza Janés. 
Duroselle, J. B. (1974). Opinion, attitude, mentalité, mythe, idéologie: essai de clarification. Relations internationales, 2, pp. 3-23.

Duroselle, J. B. (1992). Todo imperio perecerá. Buenos Aires, Fondo de Cultura Económica

Frank, R. (2012). Pour l'historoire des relations internationales. Paris, Francia: Presses Universitaires de France.

Ferrer, A. (2007). Globalización, desarrollo y densidad nacional. En Vidal, G. y Guillén R., A. (Comp). Repensar la teoría del desarrollo en un contexto de globalización. Homenaje a Celso Furtado (pp. 431-438). Buenos Aires, Argenitna: CLACSO. Recuperado de https://bit.ly/2FTzbfV.

Figallo, B. y Henríquez, M. J. (2020). "De lo internacional a lo transnacional: renovación y complejidad en la Historia de las Relaciones Internacionales. Estudos IberoAmericanos, 46 (1), pp. 1-17. Recuperado de: https://bit.ly/2RRTv3D.

Guillen, P. (2012). La puissance et le pouvoir. Facteurs internes et facteurs externes dans la vie politique. En Frank, R. (Ed.), Pour l'histoire des relations internationales (pp. 217-230). Paris, Francia: Presses Universitaires de France.

Grosser, P. (2012). L'histoire des relations internationales à l'épreuve des interactions transnationales. En Frank. R. (Ed.), Pour l'historoire des relations internationales (pp. 271-290). Paris, Francia: Presses Universitaires de France.

Haas, P. (1992). Introduction: Epistemic Communities and International Policy Coordination. International Organization, 46 (1). pp. 1-35. Recuperado de: https://bit.ly/2Hh6UAd.

Hill, C. (2003) The Changing Politics of Foreign Policy. Basingstoke: Palgrave Macmillan.

Hazleton, W. (1988). Los Procesos de Decisión y las Políticas Exteriores. En Wilhelmy, M. (Ed.), La Formación de la Política Exterior. Los Países Desarrollados u América Latina. Anuario RIAL, (pp. 15-37). Buenos Aires, Argentina: GEL.

Hudson, V. y Vore, C. (1995). Foreign Policy Analysis Yesterday, Today, and Tomorrow. Mershon International Studies Review, 39 (2), pp. 209-238.

Jessop, R. (2011). Reflections on the State, State Power, and the World Market. En Ibarra, P. y Cortina, M. (Comp.), Recuperando La Radicalidad. Un Encuentro En Torno al Análisis Político Crítico (pp. 11-27). Barcelona, España: Hacer.

Kan, J. (2015). La integración desde arriba: los empresarios argentinos frente al MERCOSUR y el $A L C A$. Buenos Aires, Argentina: Imago Mundi.

Keohane, R. y Nye, J. (1974). "Transgovernmental Relations and International Organizations". WorldPolitics, 27, pp. 39-62.

Lasagna, M. (1995). Las determinantes internas de la política exterior: un tema descuidado en la teoría de la política exterior. Estudios Internacionales, 111, pp. 387- 409.

Lasagna, M. (1996). Cambio institucional y política exterior: un modelo explicativo. Cidob d'affaires internacionales, 32, pp. 45-64.

Laufer, R. (2008). China y las clases dirigentes de América Latina: gestación y bases de una “relación especial”. Revista Mexicana de Política Exterior, 83, pp. 137-182.

Lopez Burian, C. (2015). Partidos políticos, ideología y política exterior en Uruguay (20102014). Revista Colombia Internacional, 83, pp. 135-169. 
Llenderrozas, E. y Finkielsztoyn, M. (2013). Estudios de Política Exterior: teorías, enfoques y debates. En Llenderrozas, E. (Coord.), Relaciones internacionales: teorías y debates (pp. 167-196). Buenos Aires, Argentina: Eudeba.

Luján, C. (1993). Cambio de régimen y política internacional: el caso uruguayo. Montevideo, Uruguay: División Cultura, Intendencia Municipal.

Madrid, E. (2003). Argentina-Brasil: la suma del sur. Mendoza, Argentina: Caviar Blue.

Maira, L (1988) Los escenarios internacionales y el proceso de formación de las políticas exteriores. En PNUD CEPAL, Análisis y Formulación de la política exterior, Documento de Trabajo 1 pp. 61 a 98. Recuperado de https://repositorio.cepal.org/bitstream/handle/ 11362/ 29426/S327 N962A_es.pdf

Manning, B. (1977). The Congress, the Executive and Intermestic Affairs: Three Proposals. Foreign Affairs, 55 (2), pp. 306-324.

Merke, F. (2019). Preferencias, herencias y restricciones: elementos para examinar la política exterior del Frente de Todos. Análisis Carolina, 24, pp. 1-19. Recuperado de https://bit.ly/2ZX7UjB.

Míguez, M. C. (2012). Illia y Santo Domingo: de las Columnas de Primera Plana al golpe de estado". Ciclos en la Historia, la Economía y la Sociedad, 20 (39), pp. 165-192.

Míguez, M. C. (2013). Partidos políticos y política exterior argentina. Buenos Aires, Argenitna: Ariel

Míguez, M. C. (2015). La Unión Industrial Argentina y el gobierno de Illia. Los sectores civiles y el golpe de estado de 1966. H-Industri@: Revista De Historia De La Industria, Los Servicios Y Las Empresas En América Latina, 17, pp. 64-91.

Míguez, M. C. (2016). La política exterior argentina y su vinculación con los condicionamientos internos en el siglo XXI. Revista Relaciones Internacionales, II, pp. 25-142.

Míguez, M. C. (2017). La autonomía heterodoxa y la clasificación de las políticas exteriores en la Argentina. Revista de Relaciones Internacionales, estrategia y seguridad, 12(2), pp. 207-229.

Míguez, M. C. (2018). La política exterior del tercer gobierno peronista en la Argentina (19731976). Relaciones Internacionales, 27 (55), pp. 21-49.

Míguez, M. C. (2019). Argentina y el Pacto Andino en la década de 1970: política interna y relaciones internacionales. Ciclos En la Historia, la Economía y la Sociedad, XXVI (52), pp. 33-62. Recuperado de https://bit.ly/3mzDnSF.

Míguez, M. C. y Morgenfeld, L. (2017). Política exterior y movimiento social: análisis de grandes manifestaciones frente a destacados visitantes extranjeros en la Argentina (1963-1983). Trabajos y Comunicaciones, 45, pp 1-25. Recuperado de https://bit.ly/3kFbzuf.

Mistral, J. (1978)., Compétitivité et formation de capital en longue période. Économie et statistique, 97, pp. 3-23.

Moniz Bandeira, L. A. (2006). La formación de los estados en la Cuenca del Plata: Argentina, Brasil, Uruguay, Paraguay. Brasilia, Brasil: FUNAG.

Moniz Bandeira, L. A. (2013). Brasil-Estados Unidos: a rivalidade emergente (1950-1988). Rio do Janeiro, Brasil: Civilizaçao Brasileira. 
Morgenfeld, L. (2011). Vecinos en conflicto. Argentina frente a Estados Unidos en las conferencias panamericanas (1880-1955). Buenos Aires, Argentina: Peña Lillo/Continente.

Musacchio, A. (2016). La Argentina del segundo bicentenario: imágenes de una estación desolada. Cuadernos de Economía Crítica, 4, pp. 11-38.

Musacchio, A. (2020). Neoliberalismo, inserción internacional y financiarización: una comparación entre Argentina y Portugal. Problemas Del Desarrollo. Revista Latinoamericana De Economía, 51 (201), pp. 155-178.

Nemiña, P. (2011). Del blindaje a la intransigencia: comportamiento del FMI durante la crisis económica (2000-2001). Ciclos en la Historia, la Economía y la Sociedad, 20 (39), pp. 219-243.

Osorno, G. (1995). El vínculo entre ámbitos interno e internacional. De la política de eslabones a la diplomacia de doble filo. Foro Internacional, 141, pp. 426-447.

Oszlak, O. y O’Donnell, G. (1982). Estado y políticas estatales en América Latina: Hacia una estrategia de investigación. Revista Venezolana de Desarrollo Administrativo, 1, pp. 75105.

Paradiso, J. (1994) Debates y trayectoria de la política exterior argentina, Buenos Aires: GEL.

Perina, R. (1988). El estudio de la política exterior y de las relaciones internacionales argentinas. En Perina, R. y Russell, R., Argentina En El Mundo (pp.11-18). Buenos Aires, Argentina: GEL.

Poulantzas, N. (1976). La crisis de las dictaduras. México D.F, México.: Siglo XXI.

Poulantzas, N. (1979) Estado, poder y socialismo. México D. F.: Siglo XXI.

Putnam, R. (1988). Diplomacy and Domestic Politics: The Logic of Two-Level Games. International Organization, 42 (3), pp. 427-460.

Ramírez, H. (2007). Corporaciones en el poder: institutos económicos y acción política en Brasil y Argentina : IPES, FIEL y Fundación Mediterránea. Buenos Aires, Argentina: Lenguaje Claro.

Rapoport, M. (1982). Gran Bretaña, Estados Unidos y las clases dirigentes argentinas. Buenos Aires, Argentina: Editorial de Belgrano.

Rapoport, M. (Comp.) (2016). Historia Oral de la Politica Exterior Argentina, Tomo I y II. Buenos Aires, Argentina: Octubre.

Rapoport, M. (2020.) Parece cuento que la Argentina aun exista. Buenos Aires, Argentina: Octubre.

Rapoport, M. y Madrid, E. (2011). Argentina-Brasil. De rivales a aliados. Política, economía y relaciones bilaterales. Buenos Aires, Argentina: Capital Intelectual.

Rapoport, M. y Spiguel, C. (2006). Modelos económicos, regímenes políticos y política exterior argentina. En Sombra Saraiva, J. F. (Comp.), Foreign Policy and Political Regime (pp. 131-181). Brasilia, Brasil: Instituto Brasileiro de Relaçoes Internacionais.

Reich, R. (1993). L'économie mondialisée. Paris, Francia: Dunod. 
Renouvin, P. y Duroselle, J. B. (2010). Introduction à l'histoire des relations internationals. Paris, Francia: Collin.

Rhodes, B. (2018). The World as It Is. Nueva York, Estados Unidos: Random House.

Rosenau, J. (1997). Along the domestic-foreign frontier: exploring governance in a turbulent world. Cambridge, Reino Unido: Cambridge University Press.

Russell, R. (1990). El proceso de toma de decisiones en la política exterior argentina (19761989). En Russell, R. (Comp.), Política exterior y toma de decisiones en América Latina (pp.13-59). Buenos Aires, Argentina: GEL,

Sánchez, L. (2013). Condicionantes internos de la política exterior argentina: La participación del cuerpo decisional legislativo en el diseño de la agenda internacional de la política exterior argentina (1999-2003). Tesis de posgrado. Universidad Nacional de La Plata. Facultad de Humanidades y Ciencias de la Educación. En Memoria Académica. Recuperado de: https://bit.ly/33Qej12.

Simonoff, A. (2012). Teorías en movimiento. Los orígenes disciplinares de la politica exterior y sus interpretaciones históricas. Rosario, Argentina: Prohistoria Ediciones.

Strange, S. (1988). State and markets. Londres, Reino Unido: Pinter Publishers

Sunkel, O. (1972). Big Business and 'Dependencia': A latin american view. Foreign Affairs, 50 (3), pp. 517-531.

Tausch, A. (2010). Globalisation and development: the relevance of classical "dependency" theory for the world today. International Social Science Journal, 61 (202), pp.467-488.

Thwaites Rey, M. (2005). Estado: ¿qué Estado?. En Thwaites Rey, M. y López, A. (Eds.). Entre tecnócratas globalizados y políticos clientelistas. Derrotero del ajuste neoliberal en el Estado argentino (pp. 21-41). Buenos Aires, Argentina: Prometeo.

Thwaites Rey, M. y Ouviña, H. (2016). Tensiones hegemónicas en la Argentina reciente”. En Oliver. L, El Estado ampliado en América Latina y el Caribe (pp.114-136). México D.F., México: Universidad Nacional Autónoma de México.

Tickner, A. B. (2002). Los estudios internacionales en América Latina: Subordinación intelectual o pensamiento emancipatório?. Bogotá, Colombia: Ediciones Uniandes.

Tokatlián, J. G. y Merke, F. (2014). Instituciones y actores de la política exterior como política pública. En Acuña, C. (Comp.), Dilemas del Estado Argentino. Política Exterior, económica y de infraestructura en el Siglo XXI (pp. 245-293). Buenos Aires, Argentina: Siglo XXI.

Tussie, D. (2015). Relaciones Internacionales y Economía Política Internacional: notas para el debate. Relaciones Internacionales, 48, pp. 155-175.

Van Klaveren, A. (1984). Análisis de la política exterior latinoamericana: perspectivas teóricas. En Muñoz, H. y Tulchin, J., Entre autonomía y la subordinación. Política exterior de los países de Latinoamérica (pp. 35-50). Buenos Aires, Argentina: Grupo Editorial Latinoamericano.

Van Klaveren, A. (1992). Entendiendo las políticas exteriores latinoamericanas: modelo para armar. Estudios Internacionales Santiago, 98, pp. 169-216.

Vidigal, C. (2009). Relações Brasil-Argentina. A Construção do Entendimento. 1958-1986. Curitiba, Brasil: Juruá. 
Vilar, P. (1980). Iniciación al vocabulario del análisis histórico. Barcelona, España: Crítica.

Vilar, P. (1983). Historia del Derecho. Historia 'Total'”. En Vilar, P., Economía, Derecho, Historia. Conceptos y realidades (pp. 106-139). Barcelona, España: Ariel.

Wallerstein, I. (1979). The Capitalist World-Economy. Cambridge, Reino Unido: Cambridge University Press.

Waltz, K. (1979). Theory of International Politics. Nueva York, Estados Unidos: Random House.

Zavaleta, R. (2009). El Estado en América Latina. En Tapia, L. (Comp.), La autodeterminacion de las masas (pp. 321-355). Bogotá, Colombia: Siglo del hombre/CLACSO. 